\title{
Relation of serum level of tumor necrosis factor-alpha to cognitive functions in patients with Parkinson's disease
}

\author{
Manal Mahmoud El-Kattan' ${ }^{1}$, Laila Ahmed Rashed ${ }^{2}$, Sara Refaat Shazly ${ }^{1}$ and Rania Shehata Ismail ${ }^{{ }^{*}}$
}

\begin{abstract}
Background: Inflammation is suggested to play a role in the development of non-motor Parkinson's disease (PD) symptoms. We aimed to investigate the association between serum tumor necrosis factor-alpha (TNF-a) levels and cognition in PD patients. Thirty patients with PD and 30 healthy controls were included. Evaluation and staging of PD were done using Unified PD Rating Scale. Cognitive assessment was done using Addenbrooke's Cognitive Examination (ACE-III) and trail making B tests. Measurement of serum levels of TNF-a was done.

Results: Patients had significantly worser cognitive scores than controls except for language subclass of ACE score. Mean serum TNF-a level was significantly greater in PD patients as compared to controls. TNF-a serum level was significantly negatively correlated with ACE visuospatial function. Sensitivity and specificity of TNF-a to detect cognitive dysfunction in PD using ACE III and trail making B tests were (73.1, 75\%), (57.1, 56.2\%), respectively, whereas sensitivity and specificity of TNF-a to detect severity of PD using H\&Y staging in PD were $50 \%$.
\end{abstract}

Conclusion: Patients with PD frequently have cognitive impairment. Elevated serum TNF-a levels in patients with PD, and association of this cytokine to visuospatial impairment, implicate this pro-inflammatory cytokine in the neurobiology of cognitive impairment in PD.

Keywords: Parkinson's disease, Cognitive functions, ACE-III, Trail making B test, Serum TNF-a level

\section{Background}

After Alzheimer disease, PD is the second most common neurodegenerative disorder in elderly. PD is suggested to involve interactions between genetic and environmental factors. Most cases start after the age of 50 years. The prevalence increasing to $1.5-2.5 \%$ of people above the age of 70 years [1].

PD is a heterogeneous disease with a wide range of symptoms. One classification based solely on clinical features supports two subtypes: tremor dominant PD and non-tremor dominant PD. Tremor-dominant Parkinson's disease is characterized by the absence of other motor

\footnotetext{
*Correspondence: raniashehata.neuro@yahoo.com

1 Department of Neurology, Faculty of Medicine, Cairo University, Giza, Egypt

Full list of author information is available at the end of the article
}

symptoms and a superior response to dopamine replacement therapy. A patient with non-tremor-dominant PD, on the other hand, may develop an akinetic-rigid syndrome and a postural instability problem, as well as a higher incidence of non-motor symptoms. The disease's course and prognosis differ and it is thought that the different subtypes have different pathophysiology and etiologies [2].

PD is characterized by loss of neurons and gliosis, as well as the presence of Lewy bodies (LBs). The location and distribution of neurodegeneration reflect corresponding neurological and psychiatric signs and symptoms. The aberrant aggregates of misfolded $\alpha$-synuclein are seen in LBs. The buildup of aberrant proteinaceous molecules in brain tissue is accelerated by neuroinflammation. Neurodegeneration leads to neuroinflammation, resulting in a cyclic potentiation. Many variables, 
including genetic, environmental, viral, dietary, and lifestyle factors, might trigger the proteinopathy-neuroinflammation-neurodegeneration loop [3].

Non-motor alterations, such as cognitive, autonomic and sleep disturbances are commonly encountered in PD patients. These are noteworthy determining factors of quality of life [4].

Barnum and Tansey (2012) suggested that inflammation may play a role in the development of non-motor PD symptoms [5]. Previous clinical researchers have identified possible links between these symptoms and peripheral cytokines. TNF- $\alpha$ levels in the blood have been linked to a variety of non-motor symptoms, including cognition and depression [6], while IL-6 levels have been linked to scores on the Mini-Mental State Examination (MMSE) in PD patients without dementia [7].

There is no cure or neuroprotective treatment for Parkinson's disease despite decades of research. Although there are medications that can help patients manage their symptoms and maintain their quality of life, none of them can slow or stop the degeneration of DA neurons. Alternative therapeutical techniques are being developed, and the use of PD pre-clinical models is a vital step in testing new disease-modifying strategies [8].

Chronic neuroinflammation is a common hallmark of Parkinson's disease, in which TNF- $\alpha$ expression appears to be elevated, proposing that it could be a worthy target for treatment or slowing the progression of cognitive decline in people with PD $[9,10]$.

The current work aimed to examine the possible association between serum TNF- $\alpha$ levels and cognition in PD patients.

\section{Methods}

This case-control study; included 30 PD patients and 30 age and gender matched healthy volunteers, between February 2019 and December 2019.

Written consents were taken from patients prior to participation.

Inclusion criteria: Patients with PD diagnosed according to MDS clinical diagnostic criteria for Parkinson's disease, 2015 [11]. Their age ranged between 50 and 75 years. The subjects should be educated. Mini-Mental State Examination (MMSE) score $>26$ and, Hamilton Depression Scale score $\leq 6$.

The exclusion criteria were as follows: patients with concomitant medical, metabolic or endocrinal affection such as diabetes mellitus, hepatic, renal diseases and thyroid dysfunction, patients with MRI brain showing structural lesions and presence of inflammatory or infectious diseases, which may alter serum levels of TNF- $\alpha$.

Patients were submitted to the following: clinical assessment, staging of Parkinson disease using UPDRS, cognitive assessment scales including: the Arabic version of ACE-III assesses various aspects of cognition. It is reported to have a better ability than the MMSE to detect sub-cortical dementia syndromes [12]. We used the validated Egyptian-Arabic ACE-III; it offers high sensitivity, specificity [13]. Trail making test (part B) is used to measure the information processing speed and executive functions [14] and measurement of serum level of tumor necrosis factor- $\alpha$ by enzyme-linked immune assay ELISA. It was performed at the Biochemistry department of faculty of medicine. Blood was drawn into EDTA-treated tubes and centrifuged at $4{ }^{\circ} \mathrm{C}$ for $20 \mathrm{~min}$ at $2000 \mathrm{rpm}$. TNF- $\alpha$ was measured using an in-house ELISA technique that used commercially available capture and detection antibody pairs and cytokine standards (BD Pharmingen BD OptEIATM ELISA Sets).

\section{Statistical analysis}

The collected data were coded, tabulated, and statistically analyzed using IBM SPSS statistics (Statistical Package for Social Sciences) software version 22.0, IBM Corp., Chicago, USA, 2013. Simple descriptive statistics (arithmetic mean and standard deviation) used for summary of normal quantitative data and frequencies used for qualitative data. Bivariate relationship was displayed in cross-tabulations and comparison of proportions was performed using the Chi-square and Fisher's exact tests where appropriate. Paired T-test was used to compare normally distributed quantitative data. Pearson correlation was used to compare normally distributed quantitative data. Accuracy was represented using the terms sensitivity, and specificity. Receiver operator characteristic (ROC) analysis was used to determine the optimum cut-off values. The level of significance was set at probability $(p)$ value $<0.05$.

\section{Results}

The age of patients ranged from 50 to 75 years with a mean value $63 \pm 8$. Also, the age of control subjects ranged from 50 to 75 years with a mean value $62 \pm 4$. The patients group included 25 males $(83.3 \%)$ and 5 females (16.7\%). The control group included also 25 males (83.3\%) and 5 females (16.7\%). Patients and controls were matched regarding mean age and sex distribution $(p=0.45,0.73)$, respectively.

The duration of the disease ranged from 1 to 6 years with a mean $3 \pm 1$ years. The age at onset of the disease ranged from 49 years old to 73 years with mean $55 \pm 7$ years.

UPDRS scores ranged from 23 to 77 with median 45 and mean 47. The Hoehn and Yahr scores ranged from 1 to 3 with mean $2.4 \pm 0.8$. The median was 2 . 
Table 1 Demographic and clinical data of the studied groups

\begin{tabular}{|c|c|c|c|c|}
\hline & Patients & & Control & $p$ value \\
\hline Age (year) & $63 \pm 8$ & & $62 \pm 4$ & 0.45 \\
\hline \multicolumn{5}{|l|}{ Gender } \\
\hline Male & $25(83.3 \%)$ & & $25(83.3 \%)$ & 0.73 \\
\hline Female & $5(16.7 \%)$ & & $5(16.7 \%)$ & \\
\hline Duration (year) & $3 \pm 1$ & & - & \\
\hline Age at onset of the disease & $55 \pm 7$ & & - & \\
\hline \multirow[t]{3}{*}{ Total UPDRS score } & Range & $23-77$ & - & \\
\hline & Mean & 47 & & \\
\hline & Median & 45 & & \\
\hline \multirow[t]{3}{*}{ HY scale } & Range & $1-3$ & - & \\
\hline & Mean & $2.4 \pm 0.8$ & & \\
\hline & Median & 2 & & \\
\hline \multicolumn{5}{|l|}{ Treatment } \\
\hline Dopaminergic drugs & $(22) 73.3 \%$ & & - & \\
\hline Untreated & (5) $16.7 \%$ & & & \\
\hline Non-compliant & (3) $10 \%$ & & & \\
\hline
\end{tabular}

UPDRS Unified Parkinson's Disease Rating Scale, HY Hoehn and Yahr

Table 2 Comparison between TNF a serum level between the two groups

\begin{tabular}{llll}
\hline TNF-a level (pg/ml) & Patients & Control & $\boldsymbol{p}$ value \\
\hline & $114.390 \pm 18.0341$ & $40.090 \pm 8.4638$ & $<0.001^{*}$
\end{tabular}

TNF- $a$ tumor necrosis factor-alpha

*Significant

In the current study, 22 patients (73.3\%) received treatment in the form of dopaminergic drugs including levodopa and dopamine agonists whereas, 5 patients (16.7\%) were untreated and 3 patients $(10 \%)$ were not compliant.

Demographic and clinical data of the studied groups are illustrated in Table 1.

In the patients group, TNF serum level ranged from 78.2 to $169.1 \mathrm{pg} / \mathrm{ml}$, the mean was $114.4 \mathrm{pg} /$ $\mathrm{ml} \pm 113.6 \mathrm{pg} / \mathrm{ml}$. In the control group, TNF- $\alpha$ serum level ranged from 31.5 to $64.9 \mathrm{pg} / \mathrm{ml}$ the mean was $40.1 \pm 38.2 \mathrm{pg} / \mathrm{ml}$ (Table 2).

Patients had significant worse cognitive scores than controls $(p<0.05)$ except for language subclass of ACE score $(p=0.272)$ (Table 3$)$.

Mean serum TNF level was significantly greater in PD patients as compared to controls $(p<0.001)$ (Fig. 1$)$.

TNF- $\alpha$ serum level was significantly negatively correlated with ACE visuospatial $(p=0.03, r=-0.4)$. However, no statistically significant correlation was found between serum level of TNF- $\alpha$ and ACE total, ACE attention, ACE memory, ACE verbal fluency, ACE language and trail making test (Fig. 2).
Table 3 Comparison between results of neurocognitive scales in both groups

\begin{tabular}{lccc}
\hline Mean \pm SD & $\begin{array}{l}\text { Patients group } \\
(\boldsymbol{n}=\mathbf{3 0})\end{array}$ & $\begin{array}{l}\text { Control group } \\
(\boldsymbol{n}=\mathbf{3 0})\end{array}$ & $\boldsymbol{p}$-value \\
\hline ACE-III & $81.40 \pm 5.957$ & $94.33 \pm 3.066$ & $<0.001^{* *}$ \\
Attention ACE & $17.13 \pm 0.900$ & $17.53 \pm 0.571$ & $0.044^{*}$ \\
Memory ACE & $19.63 \pm 4.115$ & $24.30 \pm 1.119$ & $<0.001^{* *}$ \\
Verbal fluency ACE & $6.87 \pm 2.609$ & $12.67 \pm 0.959$ & $<0.001^{* *}$ \\
Language ACE & $24.93 \pm 1.311$ & $25.23 \pm 0.679$ & 0.272 \\
Visuospatial ACE & $13.33 \pm 1.863$ & $15.23 \pm 0.817$ & $<0.001^{* *}$ \\
Trail making test B & $239.14 \pm 53.728$ & $103.10 \pm 24.669$ & $<0.001^{* *}$ \\
\hline
\end{tabular}

ACE-III Addenbrooke's Cognitive Examination

*Significant, ** highly significant

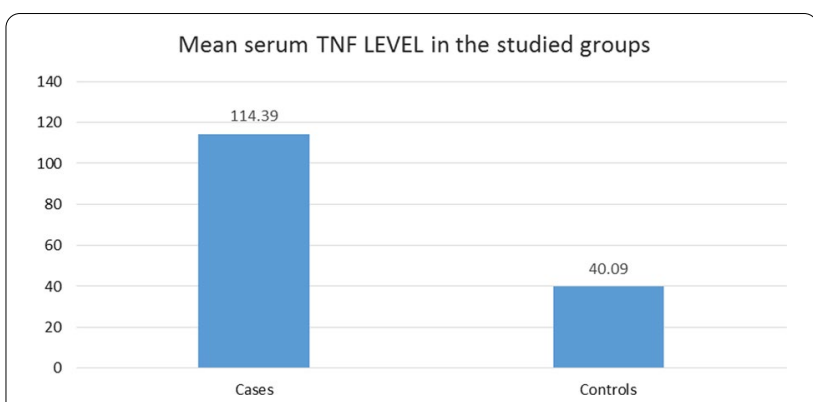

Fig. 1 Comparison of TNF-a mean serum level between the studied groups. Mean serum TNF level was significantly greater in PD patients as compared to controls $(p<0.001)$. TNF-a tumor necrosis factor-a

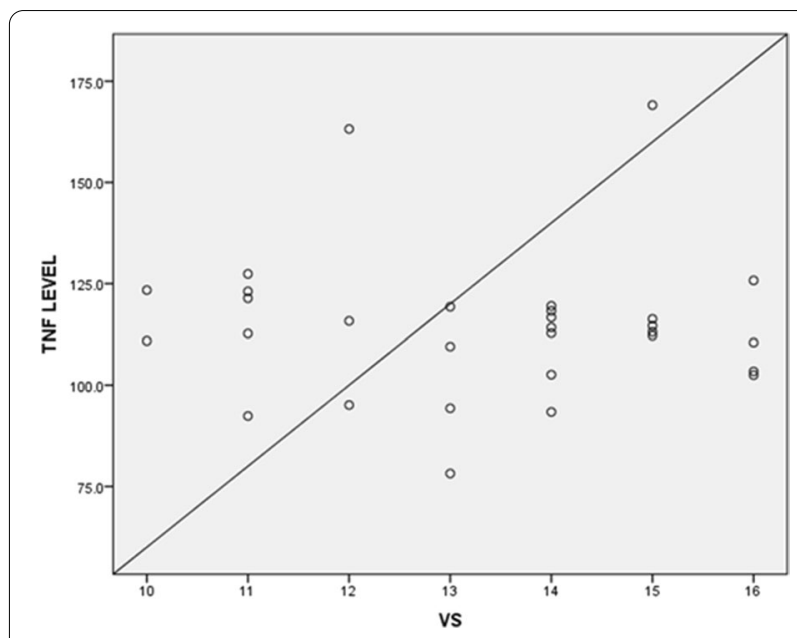

Fig. 2 Correlation between TNF-a serum level and ACE visuospatial. TNF-a serum level was significantly negatively correlated with ACE visuospatial ( $p=0.03, r=-0.4$ ). TNF- $a$ tumor necrosis factor- $a$, ACE visuospatial visuospatial subset of Addenbrooke's Cognitive Examination, VS visuospatial 
Serum levels of TNF- $\alpha$ were not significantly correlated with either mean total UPDRS or HY scores $(p>0.72$, $0.64)$, respectively.

Serum levels of TNF- $\alpha$ were not significantly with age of patients, age at onset of the disease or disease duration $(p=0.06, r=0.33),(p=0.08, r=0.32), \quad(p=0.74$, $r=-0.06)$, respectively.

ROC curve for TNF- $\alpha$ to detect cognitive impairment in patients with PD using ACE III: we observed that at cut-off point value $>110.7$, the sensitivity and specificity of TNF- $\alpha$ were $73.1,75 \%$, respectively (AUC $=0.76, p$ value $=0.08)($ Fig. 3).

ROC curve for TNF- $\alpha$ to detect cognitive impairment in patients with PD using trail making B.: at cut-off point value $>113.65$, sensitivity and specificity of TNF- $\alpha$ were $57.1,56.2 \%$, respectively $(\mathrm{AUC}=0.63, p$ value $=0.198$ ) (Fig. 4).

ROC curve for TNF- $\alpha$ to detect severity of PD using $\mathrm{H} \& \mathrm{Y}$ staging.: at cut-off point value $>113.65$, sensitivity and specificity of TNF- $\alpha$ were $50 \%(\mathrm{AUC}=0.49, p$ value $=0.69)($ Fig. 5).

\section{Discussion}

PD is often accompanied by early non-motor symptoms (NMS), which include autonomic dysfunction, psychiatric disturbances, sleep disorders, olfactory dysfunction,

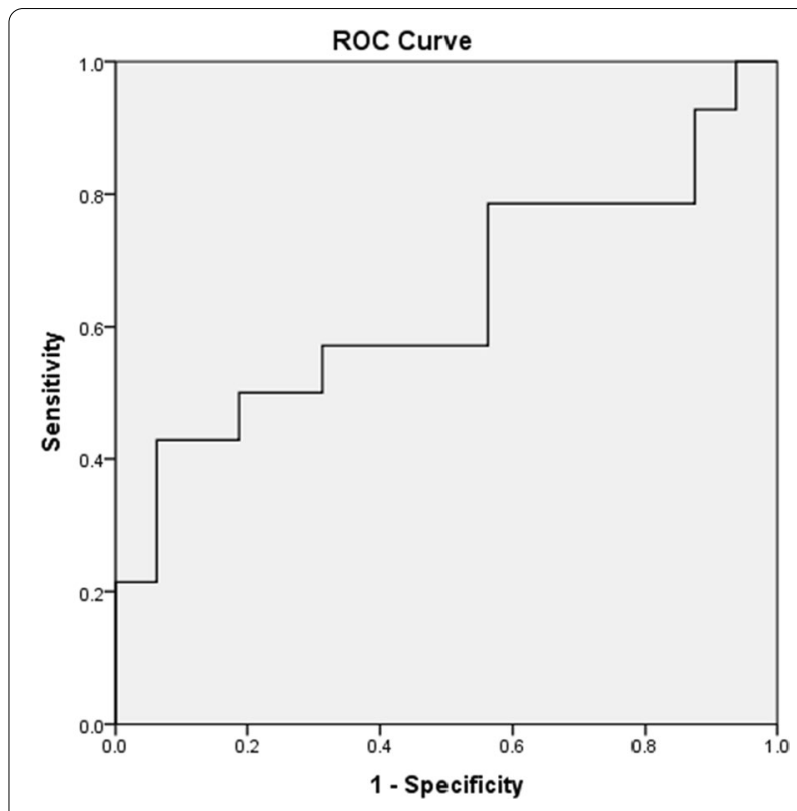

Fig. 4 ROC curve for TNF-a to detect cognitive impairment in patients with PD using trail making B. At cut-off point value $>113.65$, sensitivity and specificity of TNF-a were $57.1,56.2 \%$, respectively (AUC $=0.63, p$ value $=0.198$ ). TNF- $a$ tumor necrosis factor- $a, P D$ Parkinson's disease

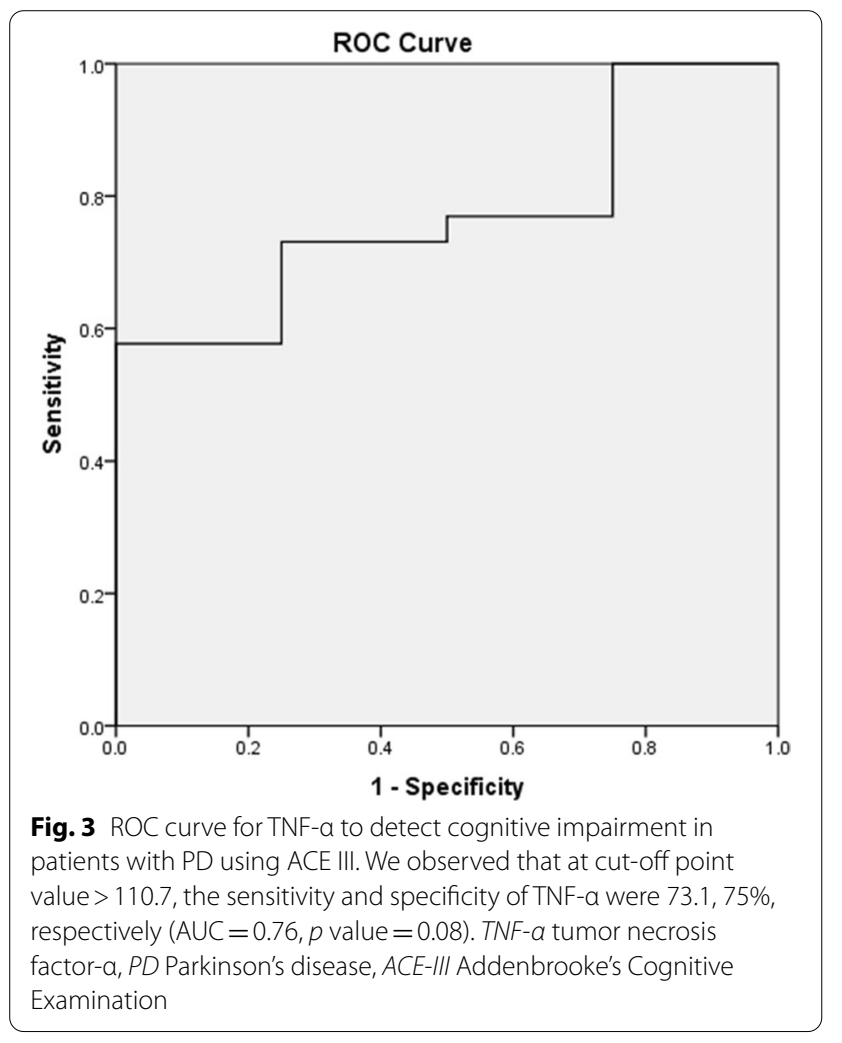

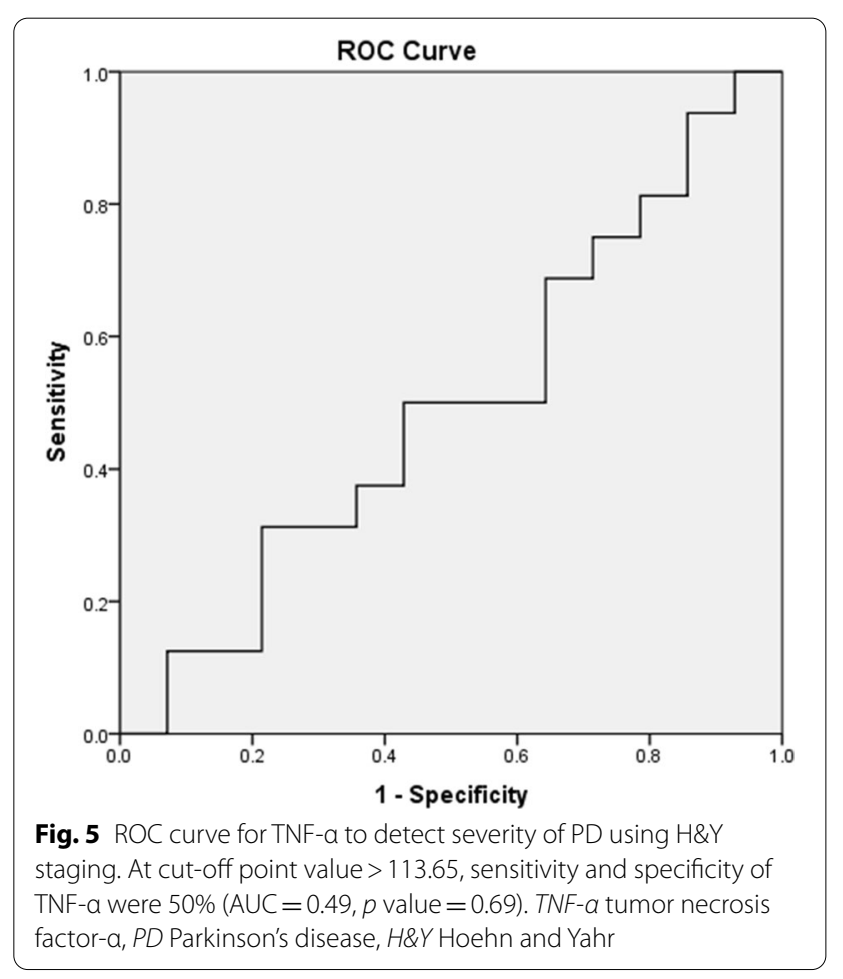


fatigue, and cognitive impairment (CI) [15]. CI has been described as the most disabling NMS in PD, and it has been shown to have a significant impact on quality of life later in the disease $[16,17]$. Furthermore, CI in PD has been linked to the onset of dementia as the disease advances [18]. As a result, CI is a significant NMS with a broad impact across the PD time frame [19].

Previous research employing bilateral intrastriatal 6-OHDA animal models consistently demonstrated significant effects on anxiety and depressive-like behaviors [20].

The present work showed that PD patients have cognitive dysfunction that includes the fields of memory, verbal fluency, visuospatial and executive functions. This was in accordance with Muslimovic and colleagues [21] who studied in detail a cohort of 115 PD patients. They detected that nearly all PD patients had executive dysfunction, $50 \%$ of patients displayed visuospatial deficits, and $45 \%$ had memory deficits compared to controls. In line with this observation, Bronnick and colleagues [22] found that patients with PD had an early impairment in free recall verbal and visual memory tests.

McKinlay and colleagues [4] stated that difficulties in planning and organizing daily life may be experienced very early by patients and neither visual recognition nor motion perception symptoms are experienced by PD patients in the non-dementia stage. In addition, Pfeiffer and colleagues [23] stated that in patients with PD, the language function appears relatively conserved while other cognitive domains are nearly equally affected, even in the early stage which was consistent with our study results.

The "dual syndrome theory" could explain this variability in the affected cognitive domains in people with PD. This hypothesis suggests that attention/working memory and executive functions are primarily affected in PD patients with more fronto-striatal network dysfunction, which is modulated by dopamine, whereas memory, language, and visuospatial functioning are primarily affected in patients with more posterior cortical degeneration, which is related to greater cholinergic loss. As a result, a thorough examination of individual cognitive domains is essential for determining the underlying pathology and, as a result, developing successful treatments [24].

Compared with the motor symptoms, little is known about the mechanisms underlying cognitive decline in PD. Inflammatory cytokines is suggested to play a key role in the neurobiology of the onset and/or maintenance of certain non-motor symptoms in Parkinson's disease [25]. Microglia are overactive in areas of the brain affected by dopaminergic loss early in Parkinson's disease (PD), and inflammation may be the cause of this selective neuronal loss [26, 27].
The current study revealed that serum TNF- $\alpha$ level was significantly greater in patients with PD compared to control group. This goes in agreement of previous studies which found that serum levels of TNF- $\alpha$ were higher in patients with PD compared to healthy subjects [28-32]. On the contrary, previous studies found that there is no statistically significant variance among PD patients and healthy controls regarding the TNF- $\alpha$ serum level [33, 34]. They explained their results by the heterogeneity of PD pathophysiology.

In PD patients, higher levels of circulating pro-inflammatory cytokines have been linked to lower cognitive performance. A study comprising 52 PD patients has found increased levels of TNF- $\alpha$ were associated with worse cognitive performance [6]. In accordance with this finding, we found a statistically significant negative correlation between visuospatial function and serum TNF- $\alpha$ level. On the other hand, some other studies failed to show any correlation between inflammatory parameters and cognitive function [35, 36]. This disparity could be explained by the lack of standardized procedures for assessing cognitive performance in people with Parkinson's disease, making comparisons between research difficult.

A considerable improvement in cognitive tests in people with PD after therapy with Etanercept, a TNF- $\alpha$ inhibitor [37], backed up the theory that pro-inflammatory cytokines are associated to cognitive changes in PD. In addition, the impairment of posterior cortical cognitive tasks is linked to early conversion to dementia, according to Williams and colleagues [38]. We found that increased TNF levels were associated with visuospatial impairment that is related to early conversion to dementia. Therefore, the preferential negative correlation of serum TNF- $\alpha$ with visuospatial function demonstrated in this study could imply that serum TNF- $\alpha$ is linked to cognitive deterioration in PD patients.

TNF- $\alpha$ is involved in synaptic transmission and plasticity [39], but it is also related to neurodegeneration and links its neurotoxic effects with the production of mitochondrial dysfunction through a mechanism involving TNF receptor 1 [40, 41]. TNF- $\alpha$ also enhances glutamatemediated cytotoxicity by increasing glutamate production in microglia due to glutaminase overexpression, inhibiting glutamate reuptake in astrocytes, and increasing the location of ionotropic receptors while decreasing the expression of GABA receptors in synapses [42-44].

In summary, we studied relationships between cognitive impairment in PD patients and TNF- $\alpha$ levels. The results showed that PD patients had significantly worse cognitive scores in trail making test and ACE except for language subclass, and the levels of TNF- $\alpha$ were significantly higher in PD patients compared to healthy 
controls, and the levels of TNF- $\alpha$ were significantly negatively correlated with the visuospatial function of ACE. We concluded that TNF- $\alpha$ pro-inflammatory cytokine is involved in the pathogenesis of cognitive impairment in PD.

The limitation of this study was the small number of enrolled subjects. A longitudinal follow-up study is essential to investigate alterations in the serum TNF- $\alpha$ profile with disease progression. Categorization of $\mathrm{PD}$ patients into 3 groups that include patients with normal cognition (PD-NC), PD patients with mild cognitive impairment (PD-MCI) and Parkinson's disease dementia (PDD_ is recommended to demonstrate serum TNF- $\alpha$ levels among these groups.

\section{Conclusion}

From all previous results, it could be concluded that patients with PD frequently have cognitive impairment. Elevated serum TNF- $\alpha$ levels in patients with PD, and association of this cytokine to visuospatial impairment, implicate this pro-inflammatory cytokine in the neurobiology of cognitive impairment in PD. Further research into these relationships could provide insights into the pathogenesis, course, and treatment of cognitive impairment in PD. In the early phases of the cognitive decline, targeting TNF-action in the brain could be a promising disease-modifying method for slowing and reducing severity of cognitive dysfunction in PD.

\section{Abbreviations}

ACE III: Addenbrooke's Cognitive Examination; Cl: Cognitive impairment; HY: Modified Hoehn-Yahr staging; MMSE: Mini-Mental State Examination; NMS: Non-motor symptoms; PD: Parkinson's disease; TNF-a: Tumor necrosis factoralpha; UPDRS: Unified PD Rating Scale.

\section{Acknowledgements}

The authors acknowledge subjects for their participation and cooperation in this study.

\section{Authors' contributions \\ MME: research idea and conception, data acquisition, data analysis and inter- pretation. LAR: performing and reviewing the laboratory workup. SRS: data acquisition, data analysis and interpretation. RSI: data acquisition, data analysis and data interpretation, and manuscript writing and reviewing. All authors have read and approved the manuscript.}

\section{Funding}

This research received no specific grant from any funding agency in the public, commercial, or not-for-profit sectors.

\section{Availability of data and materials}

The datasets generated and/or analyzed during the current study are not publicly available due to current Cairo University regulations and Egyptian legislation, but are available from the corresponding author on reasonable request and after institutional approval.

\section{Declarations}

\section{Ethics approval and consent to participate}

The aim and procedures of the study were explained to every participant and an informed written consent was obtained from all participants before being enrolled in the study. The study was approved by the ethical committee of the Neurology Department, Cairo University Hospitals. (15/1/2019).

\section{Consent for publication}

Not applicable.

\section{Competing interests}

The authors declare that they have no competing interests (financial and non-financial). We declare that the research was conducted in absence of any commercial relationships that could be constructed as a potential conflict of interest.

\section{Author details}

${ }^{1}$ Department of Neurology, Faculty of Medicine, Cairo University, Giza, Egypt. ${ }^{2}$ Department of Biochemistry, Faculty of Medicine, Cairo University, Giza, Egypt.

Received: 6 October 2021 Accepted: 5 February 2022

Published online: 19 February 2022

\section{References}

1. Rocha EM, De Miranda B, Sanders LH. Alpha-synuclein: pathology, mitochondrial dysfunction and neuroinflammation in Parkinson's disease. Neurobiol Dis. 2018;109:249-57.

2. Marras C, Chaudhuri KR. Nonmotor features of Parkinson's disease subtypes. Mov Disord. 2016;31(8):1095-102

3. Tanaka M, Toldi J, Vécsei L. Exploring the etiological links behind neurodegenerative diseases: Inflammatory cytokines and bioactive kynurenines. Int J Mol Sci. 2020;21(7):2431.

4. McKinlay A, Grace RC, Dalrymple-Alford JC, Anderson TJ, Fink J, Roger D. Neuropsychiatric problems in Parkinson's disease: comparisons between self and caregiver report. Aging Ment Health. 2008;12(5):647-53.

5. Barnum CJ, Tansey MG. Neuroinflammation and non-motor symptoms: the dark passenger of PD? Curr Neurol Neurosci Rep. 2012;12(4):350-8.

6. Menza M, Dobkin RD, Marin H, Mark MH, Gara M, Bienfait K, et al. The role of inflammatory cytokines in cognition and other non-motor symptoms of Parkinson's disease. Psychosomatics. 2010;51(6):474-9.

7. Scalzo P, Kümmer A, Cardoso F, Teixeira AL. Serum levels of interleukin-6 are elevated in patients with Parkinson's disease and correlate with physical performance. Neurosci Lett. 2010;468(1):56-8.

8. larkov A, Barreto GE, Grizzell JA, Echeverria V. Strategies for the treatment of Parkinson's disease: beyond dopamine. Front Aging Neurosci. 2020;12:4.

9. Tweedie D, Sambamurti K, Greig NH. TNF-a inhibition as a treatment strategy for neurodegenerative disorders: new drug candidates and targets. Curr Alzheimer Res. 2007;4(4):378-85.

10. Frankola AK, Greig HN, Luo W, Tweedie D. Targeting TNF-alpha to elucidate and ameliorate neuroinflammation in neurodegenerative diseases. CNS Neurol Disord Drug Targets. 2011;10(3):391-403.

11. Postuma RB, Berg D, Stern M, Poewe W, Olanow CW, Oertel W, et al MDS clinical diagnostic criteria for Parkinson's disease. Mov Disord. 2015;30(12):1591-601.

12. Galton CJ, Erzinçlioglu S, Sahakian BJ, Antoun N, Hodges JR. A comparison of the Addenbrooke's Cognitive Examination (ACE), conventional neuropsychological assessment, and simple MRI-based medial temporal lobe evaluation in the early diagnosis of Alzheimer's disease. Cogn Behav Neurol. 2005;18(3):144-50.

13. Qassem T, Khater MS, Emara T, Rasheedy D, Tawfik HM, Mohammedin AS, et al. Normative data for healthy adult performance on the EgyptianArabic Addenbrooke's Cognitive Examination III. MECP. 2015;22(1):27-36.

14. Reitan RM. Validity of the Trail Making Test as an indicator of organic brain damage. Percept Mot Skills. 1958;8(3):271-6. 
15. Poewe W. Non-motor symptoms in Parkinson's disease. Eur J Neurol. 2008;15(1):14-20. https://doi.org/10.1111/j.1468-1331.2008.02056.x.

16. Monchi $\mathrm{O}$, Hanganu A, Bellec P. Markers of cognitive decline in PD: the case for heterogeneity. Parkinson Relat Disord. 2016;24:8-14.

17. Petersen RC. Mild cognitive impairment as a diagnostic entity. J Int Med. 2004;256(3):183-94.

18. Wood KL, Myall DJ, Livingston L, Melzer TR, Pitcher TL, MacAskill MR, et al. Different PD-MCl criteria and risk of dementia in Parkinson's disease: 4-year longitudinal study. NPJ Parkinsons Dis. 2016:2(1):1-8.

19. Ghazi-Saidi L. Visuospatial and executive deficits in Parkinson's disease: a review. Acta Sci Neurol. 2020;3(4):8-26.

20. Magnard R, Vachez Y, Carcenac C, Krack P, David O, Savasta M, Boulet S, Carnicella S. What can rodent models tell us about apathy and associated neuropsychiatric symptoms in Parkinson's disease? Transl Psychiatry. 2016;6(3): e753.

21. Muslimović D, Post B, Speelman JD, Schmand B. Cognitive profile of patients with newly diagnosed Parkinson disease. Neurology. 2005;65(8):1239-45.

22. Brønnick K, Alves G, Aarsland D, Tysnes OB, Larsen JP. Verbal memory in drug-naive, newly diagnosed Parkinson's disease. The retrieval deficit hypothesis revisited. Neuropsychol. 2011;25(1):114.

23. Pfeiffer HC, Løkkegaard A, Zoetmulder M, Friberg L, Werdelin L. Cognitive impairment in early-stage non-demented Parkinson's disease patients. Acta Neurol Scand. 2014;129(5):307.

24. Kehagia AA, Barker RA, Robbins TW. Cognitive impairment in Parkinson's disease: the dual syndrome hypothesis. Neurodegener Dis. 2013:11(2):79-92.

25. Barnum CJ, Tansey MG. Neuroinflammation and non-motor symptoms: the dark passenger of Parkinson's disease? Curr Neurol Neurosci Rep. 2012;12(4):350-8.

26. Gerhard A, Pavese N, Hotton G, Turkheimer F, Es M, Hammers A, et al. In vivo imaging of microglial activation with [11C] (R)-PK11195 PET in idiopathic Parkinson's disease. Neurobiol Dis. 2006;21(2):404-12.

27. Herrera AJ, Castano A, Venero JL, Cano J, Machado A. The single intranigral injection of LPS as a new model for studying the selective effects of inflammatory reactions on dopaminergic system. Neurobiol Dis. 2000;7(4):429-47.

28. Kouchaki E, Kakhaki RD, Tamtaji OR, Dadgostar E, Behnam M, Nikoueinejad $H$, et al. Increased serum levels of TNF-a and decreased serum levels of IL-27 in patients with Parkinson disease and their correlation with disease severity. Clin Neurol Neurosurg. 2018;166:76-9.

29. Wang XM, Zhang YG, Li AL, Long ZH, Wang D, Li XX, et al. Relationship between levels of inflammatory cytokines in the peripheral blood and the severity of depression and anxiety in patients with Parkinson's disease. Eur Rev Med Pharmacol Sci. 2016;20(18):3853-6.

30. Koziorowski D, Tomasiuk R, Szlufik S, Friedman A. Inflammatory cytokines and NT-proCNP in PD patients. Cytokine. 2012;60(3):762-6.

31. Yang Y, Han C, Guo L, Guan Q. High expression of the HMGB1-TLR4 axis and its downstream signaling factors in patients with Parkinson's disease and the relationship of pathological staging. Brain Behav. 2018;8(4): e00948.

32. Chan L, Chung CC, Chen JH, Yu RC, Hong CT. Cytokine profile in plasma extracellular vesicles of PDand the association with cognitive function. Cells. 2021;10(3):604.

33. Alrafiah A, Al-Ofi E, Obaid MT, Alsomali N. Assessment of the levels of level of biomarkers of bone matrix glycoproteins and inflammatory cytokines from Saudi Parkinson patients. Biomed Res Int. 2020. https:// doi.org/10.1155/2019/2690205.

34. Lindqvist D, Kaufman E, Brundin L, Hall S, Surova Y, Hansson O. Non-motor symptoms in patients with Parkinson's disease-correlations with inflammatory cytokines in serum. PLoS ONE. 2012;7(10): e47387.

35. Dufek M, Hamanova M, Lokaj J, Goldemund D, Rektorova I, Michalkova Z, et al. Serum inflammatory biomarkers in Parkinson's disease. Parkinson Relat Disord. 2009;15:318-20.

36. Hassin-Baer S, Cohen OS, Vakil E, Molshazki N, Sela BA, Nitsan Z, et al. Is C-reactive protein level a marker of advanced motor and neuropsychiatric complications in Parkinson's disease? J Neural Transm. 2011;118(4):539-43.

37. Tobinick E, Gross H, Weinberger A, Cohen H. TNF-alpha modulation for treatment of Alzheimer's disease: a 6-month pilot study. Med Gen Med. 2006:8(2):25.
38. Williams-Gray CH, Foltynie T, Brayne CE, Robbins TW, Barker RA. Evolution of cognitive dysfunction in an incident Parkinson's disease cohort. Brain. 2007;130(7):1787-98

39. Stellwagen D, Malenka RC. Synaptic scaling mediated by glial TNF-a. Nature. 2006;440(7087):1054-9.

40. Doll DN, Rellick SL, Barr TL, Ren X, Simpkins JW. Rapid mitochondrial dysfunction mediates TNF-alpha-induced neurotoxicity. J Neurochem. 2015;132(4):443-51.

41. Islam MT. Oxidative stress and mitochondrial dysfunction-linked neurodegenerative disorders. Neurol Res. 2017;39(1):73-82.

42. Olmos G, Lladó J. Tumor necrosis factor alpha: a link between neuroinflammation and excitotoxicity. Mediators Inflamm. 2014;2014:1-12. https://doi.org/10.1155/2014/861231.

43. Takeuchi H, Jin S, Wang J, Zhang G, Kawanokuchi J, Kuno R, et al. Tumor necrosis factor-a induces neurotoxicity via glutamate release from hemichannels of activated microglia in an autocrine manner. J Biol Chem. 2006;281(30):21362-8.

44. Ye L, Huang Y, Zhao L, Li Y, Sun L, Zhou Y, et al. IL-1 $\beta$ and TNF-a induce neurotoxicity through glutamate production: a potential role for neuronal glutaminase. J Neurochem. 2013;125(6):897-908.

\section{Publisher's Note}

Springer Nature remains neutral with regard to jurisdictional claims in published maps and institutional affiliations.

\section{Submit your manuscript to a SpringerOpen ${ }^{\circ}$ journal and benefit from:}

- Convenient online submission

- Rigorous peer review

- Open access: articles freely available online

- High visibility within the field

Retaining the copyright to your article

Submit your next manuscript at springeropen.com 